\title{
Expression of baculovirus P35 prevents cell death in Drosophila
}

\author{
Bruce A. Hay, Tanya Wolff and Gerald M. Rubin \\ Howard Hughes Medical Institute and Department of Molecular and Cell Biology, University of California Berkeley, Berkeley, CA \\ 94720-3200, USA
}

\section{SUMMARY}

The baculovirus P35 protein functions to prevent apoptotic death of infected cells. We have expressed P35 in the developing embryo and eye of the fly Drosophila melanogaster. P35 eliminates most, if not all, normally occurring cell death in these tissues, as well as $\mathrm{X}$-irradiation-induced death. Excess pupal eye cells that are normally eliminated by apoptosis develop into pigment cells when their death is prevented by P35 expression. Our results suggest that one mechanism by which viruses prevent the death of the host cell is to block a cell death pathway that mediates normally occurring cell death. Identification of molecules that interact biochemically or genetically with P35 in Drosophila should provide important insights into how cell death is regulated.

Key words: baculovirus, P35, cell death, Drosophila

\section{INTRODUCTION}

Active self destruction of a cell, known as apoptosis, programmed cell death or physiological cell death, is a normal process in multicellular organisms. Cell death plays important roles in development and adult tissue homeostasis (Glucksman, 1950; Wyllie et al., 1980; Ellis et al., 1991; Raff, 1992; Vaux, 1993), selection of the immune cell repertoire (Murphy et al., 1990), protection of cells from potentially mutagenic DNA damage (Lowe et al., 1993; Clarke et al. 1993) and other potentially oncogenic stimuli (Evan et al., 1992; Bissonette et al., 1992; White and Stillman, 1987; Yonish-Rouach et al., 1991), and as a host defense against viral infection (Pilder et al., 1984; Subramanian et al., 1994; Clem and Miller, 1993).

One approach to identifying components of the cellular machinery that regulates cell death involves taking molecules that are known to be able to activate or inhibit cell death in one system, expressing them in Drosophila, in a nonessential tissue such as the eye, and asking if they perturb cell death in vivo. To the extent that the ability of the foreign proteins to manipulate cell death is evolutionarily conserved, genetic and physical interaction screens can be used to identify cellular proteins with which these foreign proteins interact. Some such interacting proteins may identify components of a Drosophila cell death pathway.

Viruses provide a rich source of proteins that perturb cell death. Cell death induced by viruses may be either necrotic or apoptotic in nature. Necrotic cell death may reflect either a nonspecific response to a viral takeover of host biosynthetic machinery or the result of cell lysis or cell fusion. In contrast, it has been suggested that apoptotic cell death following virus infection has evolved in multicellular organisms as a host strategy to prevent virus spread, wherein the host sacrifices a small number of infected cells to protect the organism as a whole (Clouston and Kerr, 1985; Martz and Howell, 1989). A predicted viral strategy to avoid this host response might be to synthesize proteins that interfere with such a host suicide pathway. In fact, a number of viruses have been shown to express proteins that function to delay or prevent host cell death (Chou and Roizman, 1992; Clem et al., 1991; Crook et al., 1993; Henderson et al., 1991, 1993; White et al., 1992; Gooding et al., 1988, 1991). Prevention of cell death has also been associated with viral persistence (Levine et al, 1993) and latency (Gregory et al., 1991).

Is the apoptotic cell death pathway activated and inhibited by viral proteins the same as that used during normal development and adult function of higher eukaryotes? Apoptosis can be induced in cells using a variety of stimuli involving different signal transduction mechanisms. These probably converge on a limited number of pathways that actually bring about killing. Viral proteins such as adenovirus P19 and baculovirus P35, which function to prevent cell death in multiple contexts (White and Stillman, 1987; Hashimoto et al., 1991; Gooding et al., 1991), likely identify functions at or downstream of these points of signal convergence. Identifying the molecules mediating these viral functions is thus likely to provide insight into the machinery mediating cell death. Toward this end, we have expressed baculovirus P35 in Drosophila tissues in which cell death is known to occur and have found that normally occurring and experimentally induced cell death is prevented by P35 expression.

\section{MATERIALS AND METHODS}

\section{Staging pupae}

Stocks were raised at $20^{\circ} \mathrm{C}$ on a standard cornmeal agar food. White prepupae (time zero) were collected and maintained for the reported times. The $w$ - stock used for $\mathrm{P}$ element transformation was used as the wild-type strain. 


\section{Detection of cell death}

Acridine orange staining was carried out as described in Spreij (1971). In situ nick translation to identify dying or dead cells based on the presence of damaged cellular DNA was carried out essentially as described in Meyaard et al. (1992). Embryos were fixed and the vitelline membranes removed as described in Hay et al. (1988). Third instar eye-antennal discs and pupal eyes were fixed similarly except that treatment with methanol was omitted. Fixed tissue was stored in $0.1 \mathrm{M} \mathrm{PO}_{4}, 0.3 \%$ Triton $\mathrm{X}-100$ at $4^{\circ} \mathrm{C}$. For nick translation reactions, tissue was rinsed in $1 \times$ nick translation buffer several times $(1 \times$ nick translation buffer $=50 \mathrm{mM}$ Tris- $\mathrm{HCl}(\mathrm{pH} 7.2), 10 \mathrm{mM} \mathrm{MgSO}_{4}, 100$ $\mu \mathrm{M}$ DTT, $50 \mu \mathrm{g} / \mathrm{ml} \mathrm{BSA}$ ). Tissue was incubated in the above buffer containing $30 \mu \mathrm{M}$ each of dATP, dGTP, dCTP, Bio-16-dUTP (Enzo Diagnostics, Syosset, NY) and $0.4 \mathrm{U} / \mu 1$ of E. coli DNA polymerase (New England Biolabs) for 90 minutes at room temperature. Following incorporation tissue was washed in $0.1 \mathrm{M} \mathrm{PO}_{4}(\mathrm{pH} 7.2)$, $0.3 \%$ Triton $\mathrm{X}-100,1 \% \mathrm{BSA}$ several times for a total of 30 minutes. To visualize the reaction product, a complex of avidin-horseradish peroxidase $(A+B$ reagents of the Vectastain $A B C$ kit, Vector labs, Burlingame, CA) was incubated with the tissue for one hour. Washes as above were followed by development of an insoluble DAB reaction product. Tissue was mounted in glycerol and photographed on a Zeiss axiophot microscope.

\section{Anti- $\beta$-galactosidase antibody staining}

Anti- $\beta$-galactosidase antibody staining of pupal eyes was carried out as described in Hay et al. (1988) using a rabbit polyclonal anti- $\beta$ galactosidase antibody (Cappel) diluted 1:5000 and preabsorbed against fixed wild-type embryos in $0.1 \mathrm{M} \mathrm{PO}_{4}(\mathrm{pH} 7.2), 0.3 \%$ Triton $\mathrm{X}-100$. A Vector anti-rabbit HRP kit was used as the secondary antibody.

\section{Cobalt sulfide staining}

Cobalt sulfide staining of larval and pupal eyes was carried out as described in Wolff and Ready (1991).

\section{BrdU labeling}

Labeling to identify cells engaged in S-phase activity was carried at 5 hour intervals, from 35 to 55 hours postpupation. Retinae were removed and placed in a $50 \mu \mathrm{g} / \mathrm{ml}$ solution of BrdU in Schneider's medium (GIBCO) for 1 hour at $25^{\circ} \mathrm{C}$. Tissues were then fixed and stained as in Winberg et al. (1992).

\section{Sectioning of adult heads}

Fixation, embedding and sectioning of adult fly heads was carried out as described in Wolff and Ready (1991).

\section{X-irradiation}

Third instar larvae were exposed to 4000 rads of X-irradiation using a Torex 120D X-ray inspection system (Astrophysics Research Corp., Long Beach, CA). Following irradiation, vials were placed at $25^{\circ} \mathrm{C}$ for 2-8 hours prior to dissection.

\section{Construction of eye expression vector}

PCR was used to amplify a fragment from construct 29-1 (Ellis et al., 1993). This fragment contains a pentamer of truncated glass-binding sites derived from the Drosophila Rh1 promoter. The primers used to amplify this pentamer and downstream TATA box sequences were ATCGATATCTAGATCTCGAG and GAACTCTGAATAGGGAATTCGGG. These primers contain XhoI and EcoRI sites, respectively, near their 3' end. PCR products (about $500 \mathrm{bp}$ ) were digested with XhoI and EcoRI and ligated into XhoI, EcoRI cut CaSpeR-hs (Pirotta, 1988), thus replacing the HSP 70 promoter and TATA box of CaSpeR-hs with the PCR product derived from construct 29-1. The vector generated is known as pGMR (glass multimer reporter). Plasmid pRS, obtained from Lois K. Miller (University of Georgia,
Athens), contains the P35 coding region from the baculovirus Autographa californica. This plasmid was digested with NruI and BglII. The fragment containing the P35 coding region was ligated into pGMR cut with $H p a \mathrm{I}$ and $B g l \mathrm{II}$. This plasmid, pGMRP35, was used to generate transgenic flies as described (Rubin and Spradling, 1982). Three transgenic lines containing a single P-element, integrated on the $\mathrm{X}$, second or third chromosome, were characterized. Similar phenotypes were seen with each line. Phenotypes shown are from the transgenic fly with a P-element on the third chromosome.

\section{Heat-shock expression of P35}

Heat-shock-induced expression of P35 during embryogenesis was carried out by crossing flies carrying glass under the control of a heatshock promoter (hs-glass) (Ellis et al., 1993) to flies carrying pGMRP35. The control cross involved crossing hs-glass-expressing flies to the $w$-stock used for P element transformation. Embryos from these crosses, kept at $25^{\circ} \mathrm{C}$, carrying both hs glass and GMRP35 or hs-glass alone, were heat shocked for 1 hour at $37^{\circ} \mathrm{C}$ between 3 and 5 hours after egg laying. They were allowed to develop for 5 hours and then fixed and processed as described above.

\section{RESULTS}

\section{P35 prevents cell death during Drosophila embryogenesis}

During normal Drosophila embryogenesis, large amounts of cell death are first apparent during germ band shortening and cell death continues throughout embryogenesis (CamposOrtega and Hartenstein, 1985; Abrams et al., 1993). We have examined the effects of a heat-shock-induced pulse of P35 expression on the first cell deaths in the embryo, during germband shortening. These experiments do not attempt to distinguish between a role for P35 in preventing initiation of cell death or in blocking subsequent events. Heat-shock expression of P35 several hours prior to the beginning of germ band shortening results in a loss of most cell death normally seen during early- and mid-germ band shortening (Fig. 1A-D). The identity of the cells that normally die is unknown, as is the fate of these cells following their rescue by P35. Therefore the role that cell death plays in embryogenesis is unclear. Understanding this role will be best addressed by expressing P35 in specific tissues and following the fate of cells identified using tissue-specific markers. Such an analysis is presented below for the developing eye.

\section{P35 prevents normally occurring cell death throughout eye development}

The developing Drosophila eye is made up of cells arranged in ordered, repeating units, known as ommatidia. Pattern formation is initiated in the 3 rd larval instar as a wave of morphogenesis sweeps across the eye from posterior to anterior (Wolff and Ready, 1993). The wave front is identified by a dorsoventral indentation, the morphogenetic furrow. Ommatidia are initiated at spaced intervals in the furrow and grow by accretion as neighboring cells are recruited into specific cell fates. By 30 hours of pupal life, ommatidia consist of 8 photoreceptors, 4 cone cells and 2 primary pigment cells. Unpatterned and undifferentiated cells are present between ommatidia. These cells will ultimately resolve into a honeycomb lattice of shared bristles and secondary and tertiary pigment cells. The interommatidial cell population initially contains 2 to 3 excess cells per ommatid- 

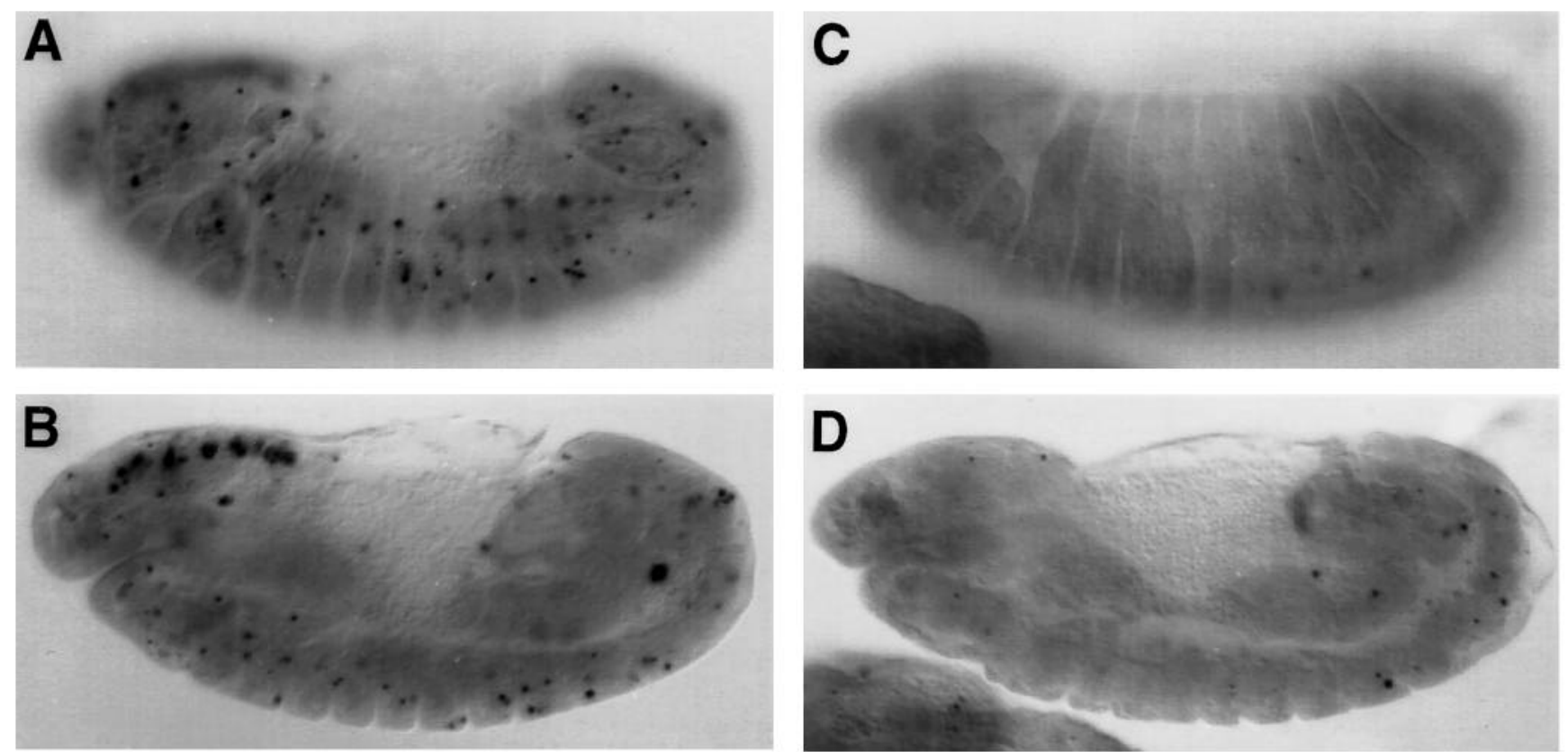

Fig. 1. Heat-shock expression of P35 during embryogenesis prevents normally occurring cell death. Flies carrying a hs-glass construct were crossed to wild-type $w$-flies or to flies carrying pGMRP35. Embryos from these crosses were processed for heat shock and in situ nick translation as described in Methods. Similar results were obtained using acridine orange with living embryos (not shown). (A) View of the lateral surface of a wild-type embryo in which expression of hs-glass had been induced. Many cells and cell fragments with damaged DNA are visible as dark dots. (B) Internal view of the same wild-type embryo. (C) Lateral surface of heat-shocked embryo transheterozygous for hsglass and GMRP35. Very few cells with damaged DNA are present in the lateral epidermis. (D) An internal view of the embryo shown in C. A few staining fragments are present.

ium. These cells are eliminated by death (Cagan and Ready, 1989; Wolff and Ready, 1991).

In order to express P35 specifically in the developing eye, an expression vector was constructed in which multimerized glass-binding sites from the Drosophila Rh1 promoter were exchanged for the heat-shock promoter in the expression vector CaSpeR-hs (see methods). Glass is expressed in all cells in and posterior to the morphogenetic furrow in the eye disc during larval development (Moses et al., 1991; Ellis et al., 1993) and at detectable levels in all retinal cells except the cone cells, during pupal development (Ellis et al., 1993). Expression is largely restricted to the developing eye, the larval photoreceptor organs and a small number of cells in the larval brain which may also be involved in photoreception (Ellis et al., 1993). Coding regions expressed downstream of these binding sites are expressed in a similar pattern (Ellis et al., 1993; B. H., unpublished). Some transgenic lines show low level expression in tissues other than the eye or show dominant phenotypes indicative of expression elsewhere (B. H., unpublished). This expression may reflect a general leakiness of the promoter or enhancer trapping by transcriptional regulatory elements in the nearby genomic region.
During eye development, cell death is conspicuous at several different stages. In the third instar eye disc, a small, variable amount of cell death is present posterior to the morphogenetic furrow (Spreij, 1971; Wolff and Ready, 1991; Bonini et al., 1993). Between 35 and 50 hours postpupation, approximately
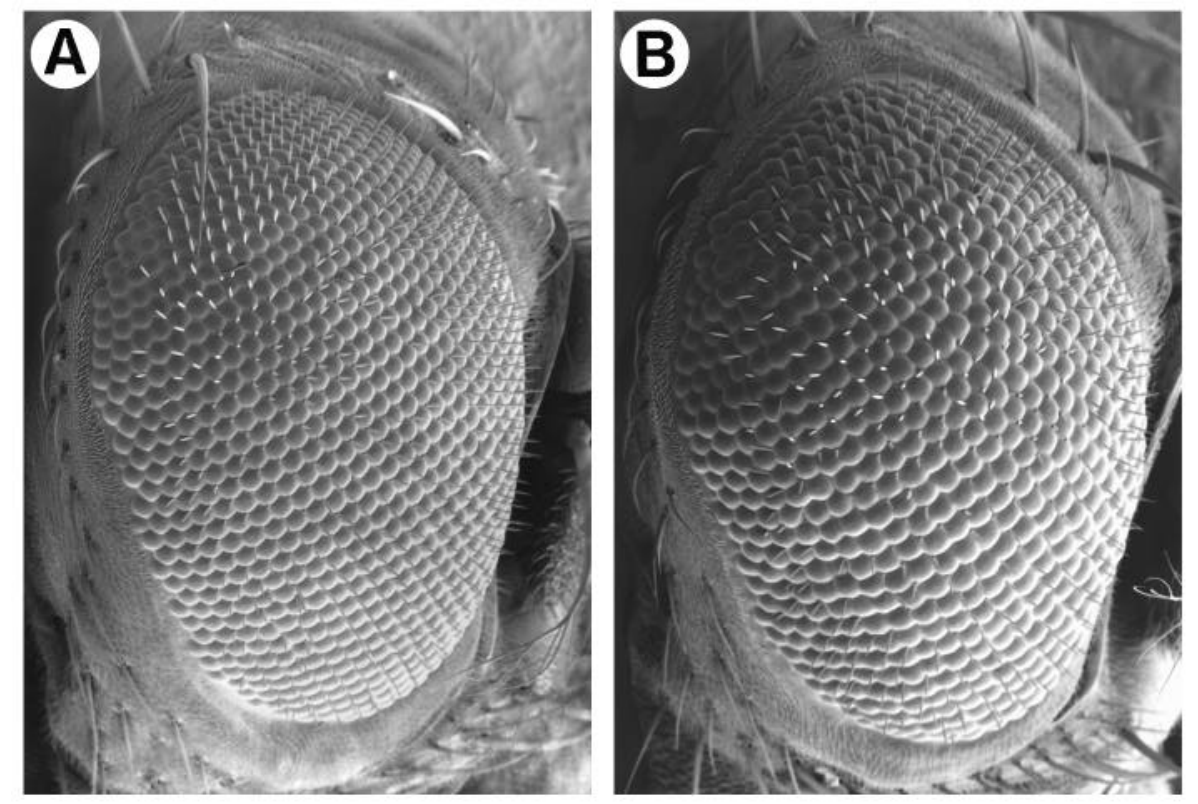

Fig. 2. Scanning electron micrographs of adult eyes from wild-type (A) and GMRP35expressing flies (B). Note that the normally highly regular array of facets, as seen in the eye in $\mathrm{A}$, is slightly disordered in the eye shown in B. 

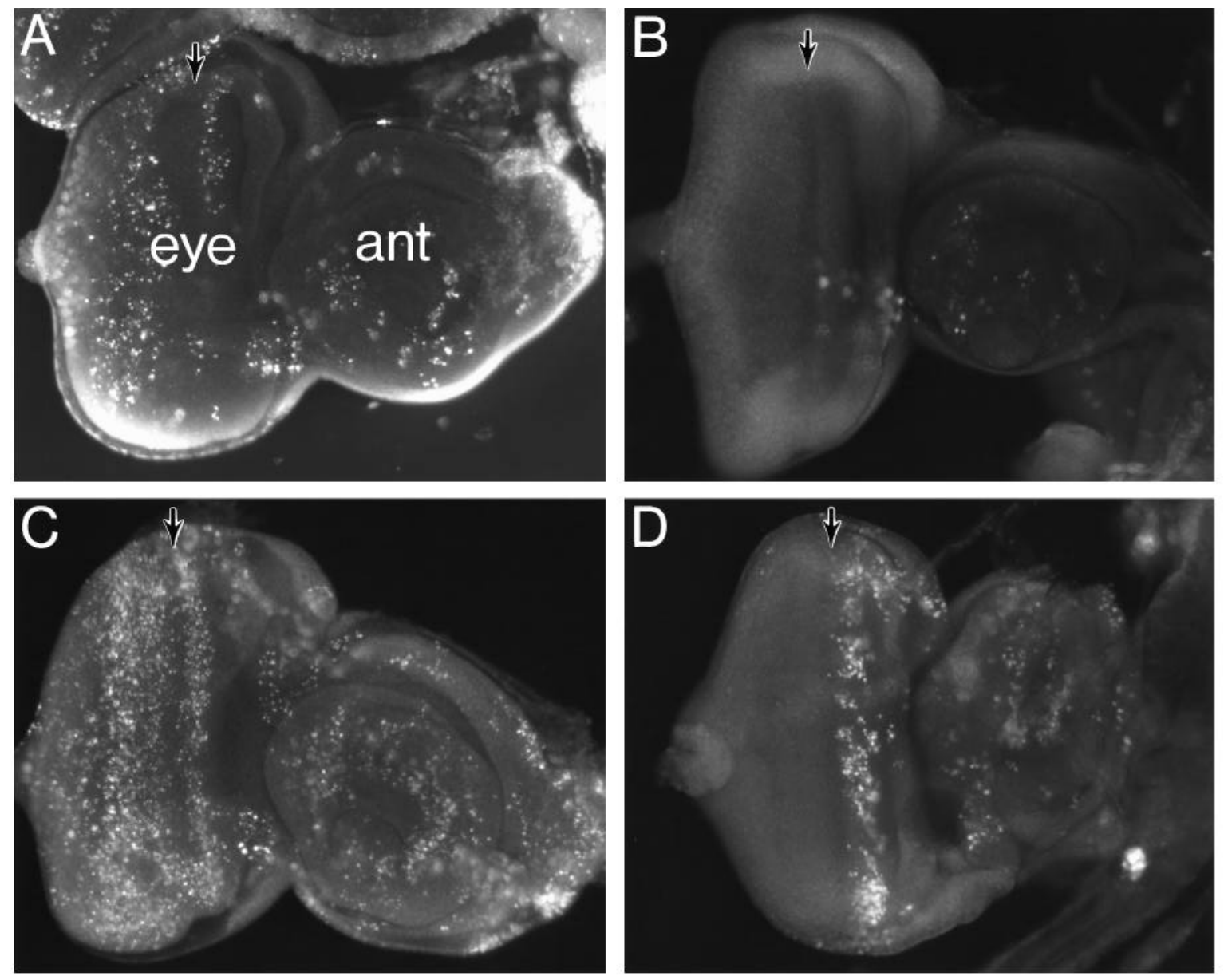

Fig. 3. P35 expression prevents normally occurring cell death and death in response to X-irradiation in the third instar eye disc. (A) Wild-type third instar eye-antennal disc stained with acridine orange. Within the eye disc (eye) a small number of dying cells are seen anterior to the morphogenetic furrow (arrow); more cell death is seen posterior to the furrow. Cell death is also observed in the antennal disc (ant). (B) Third instar eye-antennal disc expressing P35 in and posterior to the morphogenetic furrow. Staining fragments are essentially absent posterior to the morphogenetic furrow. Staining fragments are present, however, in the antennal disc, where P35 is not expressed. (C) Wild-type third instar eye disc irradiated with 4000 rads 6 hours prior to dissection. Large amounts of cell death are present anterior and posterior to the morphogenetic furrow as well as in the antennal disc. (D) P35-expressing third instar eye-antennal disc treated as in C. Large amounts of cell death are present anterior to the morphogenetic furrow and in the antennal disc. Only a few staining fragments are present posterior to the morphogenetic furrow, where P35 is expressed at high levels. In all discs anterior is to the right.

1500-2000 surplus cells are eliminated by cell death, resulting in the formation of an ordered cellular lattice (Cagan and Ready, 1989). A failure of cell death to remove these surplus cells disorganizes the lattice. In a third phase of cell death between 60 and 70 hours postpupation, the perimeter clusters, a population of stunted ommatidia that contain photoreceptors, cone cells and primary pigment cells, and that end each ommatidial row, are removed (Wolff and Ready, 1991).

The adult eye of P35-expressing flies is mildly disordered compared to that of wild type (Fig. 2). To determine if P35 expression prevents cell death, eyes were examined using acridine orange or in situ nick translation to identify dying cells, and cobalt sulfide staining to show apical profiles of cells in the epithelium. In wild-type third instar eye-antennal discs, cell death is seen in the antennal region and both anterior and posterior to the morphogenetic furrow (Fig. 3A). In discs from $\mathrm{P} 35$-expressing flies, death posterior to the furrow is eliminated (Fig. 3B); however, cell death anterior to the furrow, where glass is not detectably expressed, is still present, though often at reduced levels (Fig. 3B). This reduced death may reflect low-level expression of P35 anterior to the morphogenetic furrow. Dying cells in wild-type third instar eye discs derive from the undifferentiated epithelial cells surrounding the developing ommatidial clusters. The ultimate fate of cells prevented from dying at this stage is not known since there is no way of distinguishing between unpatterned cells that would not normally have died and those that were prevented from dying by P35 expression.

By 55 hours postpupation cell death has eliminated excess cells such that the eye shows an exact, stereotyped arrangement of cell types: four central cone cells are surrounded by two primary pigment cells; six secondary pigment cells are arranged along the faces of the ommatidial hexagon; these are separated by alternating tertiary pigment cells and bristles at the vertices of the hexagon (Fig. 4A). Perimeter clusters, which have not yet died, are also apparent (arrowhead). Similarly aged P35-expressing pupal eyes show many extra cells in the positions of secondary and tertiary pigment cells. (Fig. 4B). By 

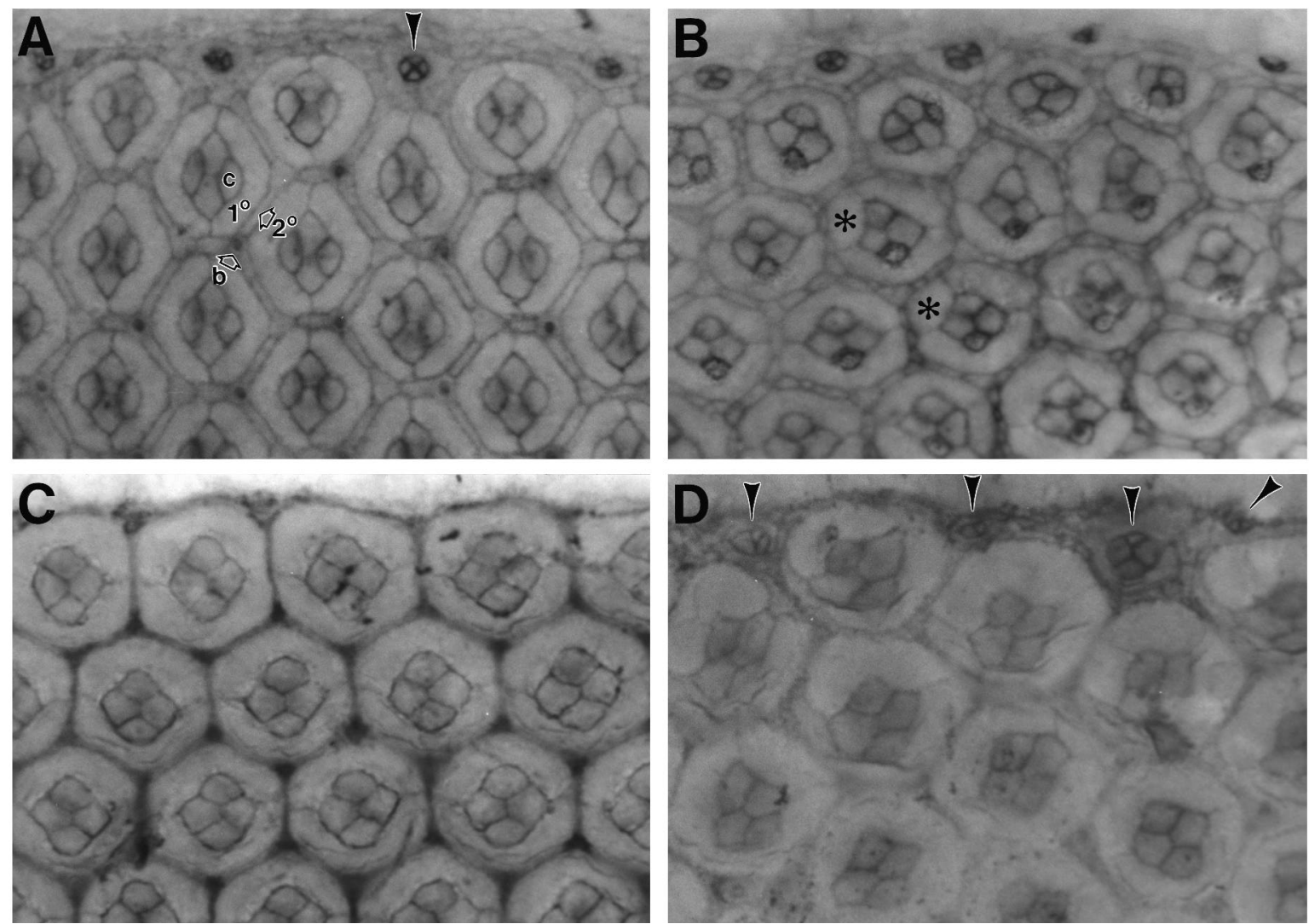

Fig. 4. Cobalt sulfide stain of wild-type and P35-expressing pupal eyes. (A) Apical surface of wild-type 55 hour pupal eye. Death has removed extra pigment cells, resulting in an ordered hexagonal array in the center of the eye. Perimeter clusters are apparent at the edge of the eye (arrow). (B) P35-expressing 55 hour pupal eye. Many extra apical profiles in the positions of secondary and tertiary pigment cells are present. Asterisks identify examples of ommatidia that contain an extra primary pigment cell. (C) Wild-type 70 hour pupal eye. Note that perimeter clusters are absent. Apical profiles of secondary and tertiary pigment cells are not visible. (D) P35-expressing 70 hour pupal eye. Perimeter clusters (arrowheads) are still present.

70 hours primary pigment cells have forced the apical surfaces of secondary and tertiary pigment cells into thin profiles so they are not visible in cobalt stains. Perimeter clusters are not present in wild-type 70 hour eyes (Fig. 4C), but are still present in 70 hour P35-expressing flies (Fig. 4D, arrowheads). Perimeter ommatidia in $\mathrm{P} 35$-expressing flies persist into adult life as seen in scanning electron micrographs and light microscope sections (Fig. 5). In wild-type adult eyes, all lenses are of uniform size and they abut the edge of the eye (Fig. 5A,B). In contrast, in eyes of P35-expressing flies small bumps, presumably reflecting a small amount of lens secretion by the perimeter clusters, are present at the end of many rows, and rows end some distance from the edge of the eye. (Fig. 5G,H). Small peripheral lenses are seen in thick sections from P35expressing eyes (Fig. 5I,J); these small profiles are absent in wild-type eyes (Fig. 5C,D). Serial sections through the periphery of P35-expressing eyes show that photoreceptor clusters are present beneath the small perimeter lens bumps (Fig. 5K,L).

In wild-type 25 hour pupal eyes, extra potential primary pigment cells are present but are ultimately excluded from contact with the cone cells and are presumed to fall back into the undifferentiated cell population (Cagan and Ready, 1989). Pupal eyes of P35-expressing flies often fail to exclude these extra primary pigment cells (Fig. 4B). We do not understand the mecha- nism behind the effect of P35 on primary pigment cell sorting, which does not appear to involve cell death (Cagan and Ready, 1989; Wolff and Ready, 1991). It is formally possible that P35's ability to prevent the death of potential secondary and tertiary pigment cells is an indirect effect of the supernumerary primary pigment cells (and thus the information that neighboring potential secondaries and tertiaries receive); however, given P35's ability to prevent death in diverse situations in Drosophila, we think this possibility is unlikely.

The excess cells in 55 hour P35 pupal eyes may result from increased cell division, prevention of cell death or some combination of the two. Acridine orange staining of living tissue or in situ nick translation on fixed tissue was carried out to determine if cell death is prevented in the eyes of P35-expressing flies. In wild-type 50 hour pupal eyes, many acridine orange-stained fragments of dying or dead cells are present throughout the eye (Fig. 6A). Staining fragments are completely absent in similarly staged eyes from P35-expressing flies (Fig. 6B). In wild-type 65 hour eyes, strong acridine orange staining is present at the perimeter, reflecting the death of the perimeter clusters (Fig. 6C). Similar staining is not present in 65 hour eyes from P35-expressing flies (Fig. 6D). These observations indicate that P35 prevents cell death throughout eye development. Extra cell divisions during pupal eye development do not contribute to the population of excess 

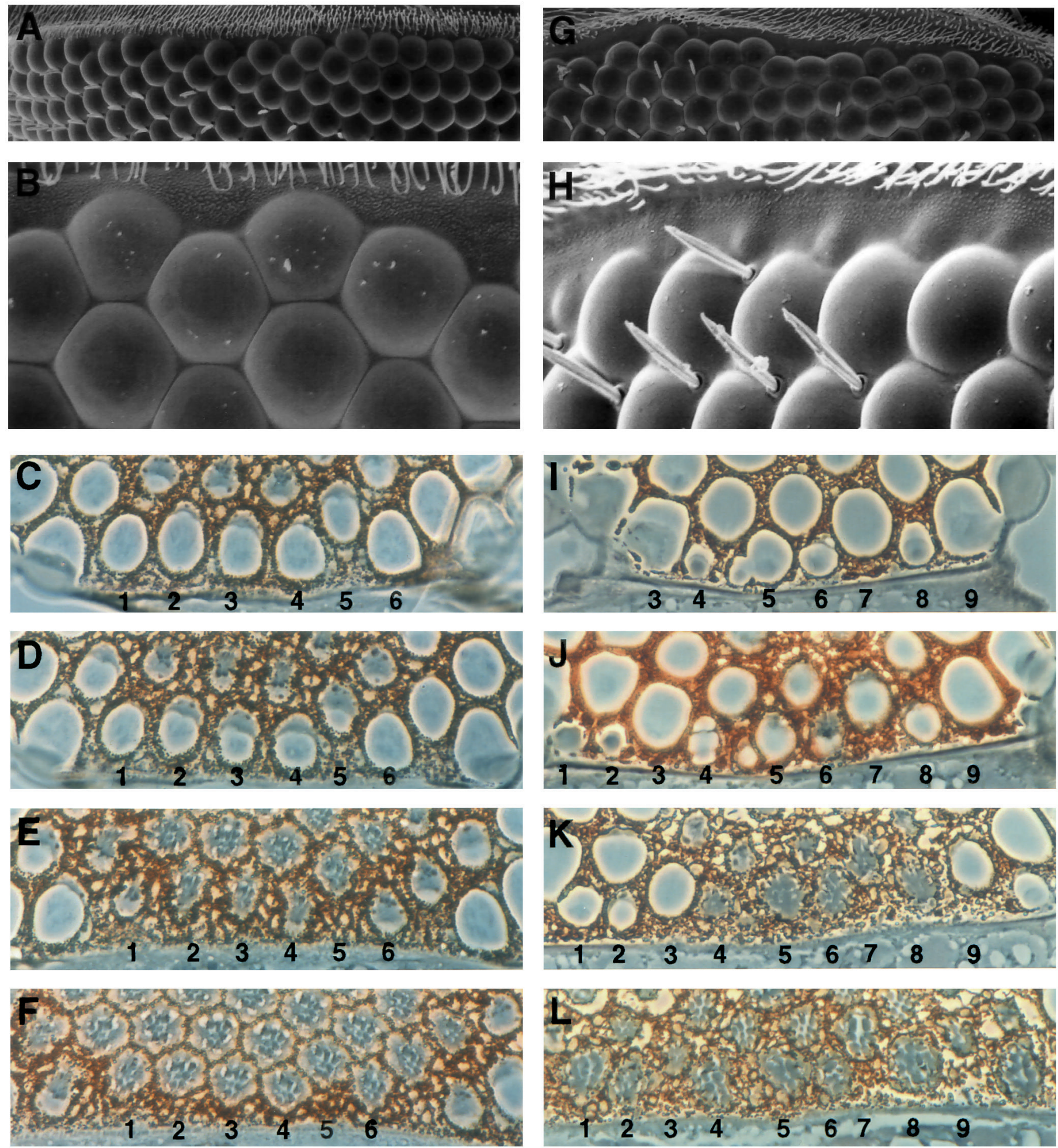

Fig. 5. The perimeter clusters are present in eyes expressing P35. Scanning electron micrographs of the edge of wild-type (A,B) and P35expressing $(\mathrm{G}, \mathrm{H})$ eyes. In $\mathrm{A}$ and $\mathrm{G}$, the edge of the eye is shown at low magnification. Note that in wild-type eyes large lenses abut the edge of the eye, whereas in the P35-expressing eye they sit some distance back. At higher magnification, small bumps (the perimeter clusters) are evident at the ends of some rows in P35-expressing eyes $(\mathrm{H})$ but not wild-type eyes $(\mathrm{B})$. Serial sections of the perimeter of wild-type (C-F) and P35-expressing eyes (I-L). Sections progress from apical (C,I) to basal (F,L). Numbers identify specific ommatidia through the depth of the sections. In the wild-type eye, the lenses are uniformly large and regularly spaced along the perimeter of the eye (C,D). Due to the curvature of the eye the lenses appear larger on the edges of the sections. Photoreceptors are seen underneath these perimeter lenses in more basal sections (E,F). In contrast, in P35-expressing eyes small lens profiles are present in apical sections at the end of rows numbered 1, 2, 4, 6 and 8 (I,J). More basal sections show that photoreceptors are present beneath these small lenses (K,L). 

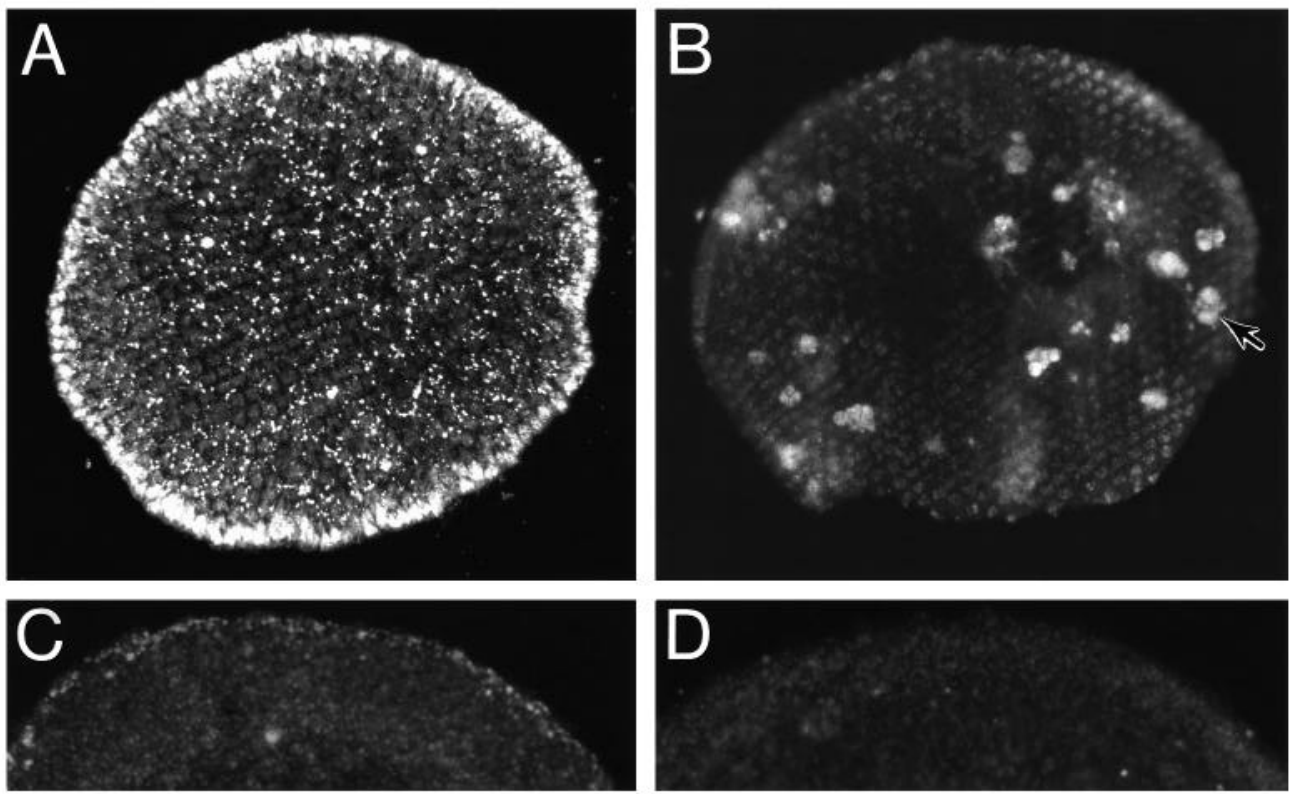

Fig. 6. Acridine orange stain of wild-type and P35-expressing pupal eyes. (A) 50 hour wild-type pupal eye. Large numbers of small, fluorescent fragments are present throughout the eye. Fluorescence at the edge of the eye is background staining. (B) 50 hour P35expressing pupal eye. Acridinestaining cell fragments are largely absent. Large diffuse staining bodies are cells of the optic lamina and fat bodies (arrow). (C) Edge of a 65 hour wild-type pupal eye. Staining fragments are present along the eye perimeter. (D) Edge of a 65 hour P35-expressing pupal eye. Staining fragments are absent from the eye perimeter. pigment cells in P35-expressing eyes since BrdU labeling of pupae from 35 to 55 hours does not label any cells other than a few bristle precursors (data not shown), which also label in wild type (Cagan and Ready, 1989).
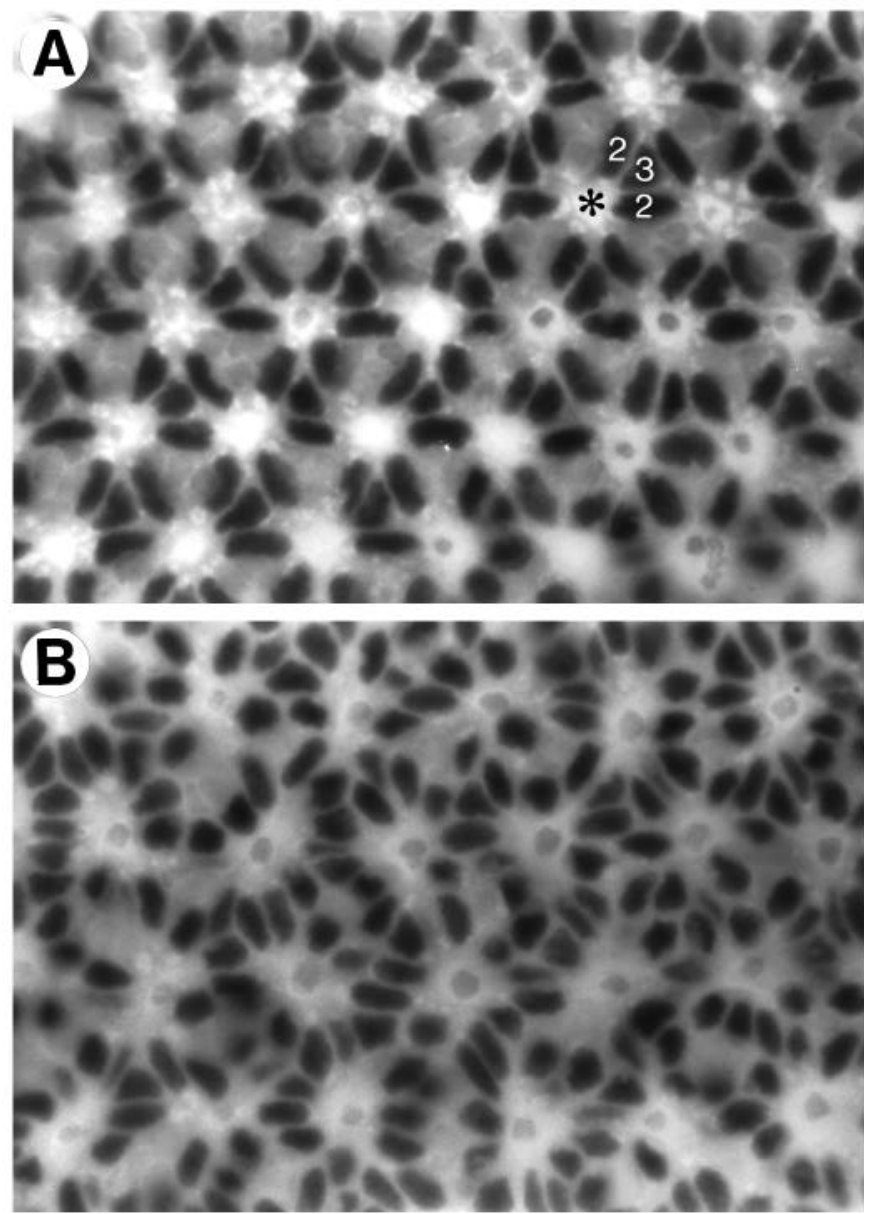

\section{Pigment cell precursors prevented from dying differentiate as pigment cells}

We determined the fate of the potential secondary and tertiary pigment cells that do not die in P35-expressing eyes with the use of a lacZ enhancer trap line (LE07). In this line, expression of lac $Z$ in the eye is restricted to secondary and tertiary pigment cells in pupae and adults (Ross Cagan, personal communication). Wild-type flies expressing LE07 have a characteristic lac $Z$ expression pattern in the midpupal eye in which three groups of three pigment cells (secondary, tertiary, secondary) are separated by unstained bristle groups (Fig. 7A). Eyes of flies expressing both P35 and LE07 have many extra staining cells (Fig. 7B), which persist into adulthood (data not shown). By these criteria, cells prevented from dying as a result of P35 expression go on to differentiate as secondary and tertiary pigment cells.

\section{P35 prevents death in response to X-irradiation}

Death of Drosophila cells in response to X-irradiation, which results in damage to DNA as well as proteins and lipids, is well documented (Ashburner, 1989), though little is known about signals required for bringing about this death (Abrams et al., 1993). To determine if P35 could prevent radiation-induced death in the Drosophila eye, wild-type and P35-expressing 3rd instar larvae were irradiated and allowed to recover from 2 to 10 hours before discs were stained with acridine orange. Acridine orange staining is greatly increased in irradiated wildtype discs (Fig. 3C) compared to unirradiated controls (Fig.

Fig. 7. Expression of $\beta$-galactosidase in enhancer trap line (LEO7) in wild-type and P35-expressing 75 hour pupal eyes. $\beta$-galactosidase is localized to nuclei of secondary and tertiary pigment cells.

Photographs focus on the pigment cell nuclear layer. (A) Expression of LEO7 in a wild-type 75 hour pupal eye is restricted to groups of three staining cells $(2,3,2)$ separated by non-staining bristle cells. These cells surround a central non-staining region (asterisk) from which photoreceptor axons protrude. (B) Expression of LEO7 in P35-expressing 75 hour pupal eyes. Many extra staining nuclei surround the central axonal region. 
3A). P35 prevents cell death posterior to the morphogenetic furrow in third instar irradiated discs (Fig. 3D). Anterior to the morphogenetic furrow and in the antennal region, where P35 is not expressed at high levels, large numbers of acridine orange-staining fragments are present.

\section{DISCUSSION}

Cell death occurs in many contexts in the Drosophila life cycle, during development as well as in the adult (Bate and Arias, 1993). Presumably, diverse signaling mechanisms are used to initiate cell death in these contexts and during the lepidotperan host cell response to baculovirus infection, yet cell death in response to these signals can be blocked through P35 expression. The ability to block death in these different contexts suggests that P35 is functioning in the cell death pathway itself, rather than changing cell fate such that cells no longer see death as an option. The above observations also suggest that cell death pathways activated during normal development, by X-ray damage, and as a response to baculovirus infection are the same or contain common components, and that one or more of these are the targets of P35 action. We cannot, however, formally exclude the possibility that P35 expression functions primarily to activate a parallel pathway which functions to antagonize cell death cascades. P35's role in preventing cell death in Drosophila is not understood; however, direct observation of baculovirus-infected cells suggests that P35 may function downstream of cell death initiation (Crook et al., 1993).

A previous screen identified two genes, roughest (rst) and echinus (ec), as being required for the normal elimination of cells from the retinal epithelium. In both these mutants, potential secondary and tertiary pigment cells that would normally die fail to do so, though perimeter clusters die on schedule (Wolff and Ready, 1991). The cellular phenotype of ec midpupal eyes is similar to that of flies expressing P35 in that secondary pigment cells are often duplicated end-to-end and ommatidia sometimes contain extra primary pigment cells. This similarity may reflect the existence of common elements in the signal transduction pathways mediating these processes, in elements targeted by P35 and in $e c$ mutants.

In the developing eye, cells prevented from dying through P35 expression either maintain their previously attained fate (the cells of perimeter clusters) or they achieve the normal cell fate alternative to death (the secondary and tertiary pigment cell precursors). Thus, cell death is not simply the cell fate resulting from a failure to maintain or receive appropriate differentiation signals; the information necessary to determine or maintain cell fate decisions is independent of that required for the decision to die. Similar observations have been made in both C. elegans embryos (Avery and Horvitz, 1987) and mammalian hemopoietic stem cells in which normally occurring cell death has been blocked (Fairbairn et al., 1993). Thus, signals that regulate cell death may, in many cases, function in pathways parallel to those required for bringing about or maintaining cell fate.

P35 expression can also prevent cell death in the nematode C. elegans (Sugimoto et al., 1994) and in mammalian neural cell cultures (Rabizadeh et al., 1993). These observations all point strongly to the conclusion that P35 targets component(s) of an evolutionarily conserved cell death pathway. The ability to express P35 in specific tissues and at specific times (this work, Brand and Perrimon, 1993) provides a powerful tool for exploring the role that cell death plays in the biology of Drosophila. P35-dependent dominant phenotypes identified as a result of preventing cell death in specific tissues should provide a useful background for genetic screens to identify interacting Drosophila proteins.

We would like to thank Lois K. Miller for providing the plasmid containing P35, Ross Cagan for providing the enhancer trap line LE07 and Mike Ellis and Betsy O'Neill for discussions concerning the expression of glass and manipulation of the multimerized glassbinding sites. We would also like to thank Ross Cagan, Donald Ready and Yuh Nung Jan for useful comments on the manuscript and Wilfred Bentham for assistance with scanning electron microscopy. This work has been supported by the Helen Hay Whitney Foundation (B. A. H.) and the American Cancer Society (T. W.); G. M. R. is a Howard Hughes Medical Institute investigator.

\section{REFERENCES}

Abrams, J. K., White, K., Fessler, L. I. and Steller, H. (1993). Programmed cell death during Drosophila embryogenesis. Development 117, 29-43.

Ashburner, M. (1989). Drosophila, a Laboratory Handbook. Cold Spring Harbor Laboratory Press.

Avery, L. and Horvitz, H. R. (1987). A cell that dies during wild-type $C$. elegans development can function as a neuron in a ced-3 mutant. Cell 51, 1071-1078.

Bate, M. and Martinez Arias, A. eds (1993). The Development of Drosophila melanogaster. Cold Spring Harbor Laboratory Press.

Bissonette, R. P., Echeverri, F., Mahboubi, A. and Green, D. R. (1992). Apoptotic cell death induced by c-myc inhibited by bcl-2. Nature $\mathbf{3 5 9}, 552$ 554.

Bonini, N. M., Leiserson, W. M., and Benzer, S. (1993). The eyes absent gene: genetic control of cell survival and differentiation in the developing Drosophila eye. Cell 72, 379-395.

Brand, A. H. and Perrimon, N. (1993). Targeted gene expression as a means of altering cell fates and generating dominant phenotypes. Development 118, 401-415.

Cagan, R. L. and Ready, D. F. (1989). The emergence of order in the Drosophila pupal retina. Dev. Biol. 136, 346-362.

Campos-Ortega, J. A. and Hartenstein, V. (1985). The Embryonic Development of Drosophila melanogaster. New York: Springer-Verlag.

Chou, J. and Roizman, B. (1992). The y134. 5 gene of herpes simplex virus 1 precludes neuroblastoma cells from triggering total shutoff of protein synthesis characteristic of programmed cell death in neuronal cells. Proc. Natl. Acad. Sci. USA 89, 3266-3270.

Clarke, A. R., Purdie, C. A., Harrison, D. J., Morris, R. G., Bird, C. C., Hooper, M. L. and Wyllie, A. H. (1993). Thymocyte apoptosis induced by p53-dependent and independent pathways. Nature 362, 849-852.

Clem, R. J., Fechheimer, M. and Miller, L. K. (1991). Prevention of apoptosis by a baculovirus gene during infection of insect cells. Science $\mathbf{2 5 4}$, 1388-1390.

Clem, R. J. and Miller, L. K. (1993). Apoptosis reduces both the in vitro replication and the in vivo infectivity of a baculovirus. J. Virol. 67, 37303738 .

Clouston, W. M. and Kerr, J. F. R. (1985). Apoptosis, lymphocytotoxicity and the containment of viral infections. Med. Hypoth. 18, 399-404.

Crook, N. E., Clem, R. J. and Miller, L. K. (1993). An apoptosis-inhibiting baculovirus gene with a zinc finger-like motif. J. Virol. 67, 2168-2174.

Ellis, R. E., Yuan, J., and Horvitz, H. R. (1991). Mechanisms and functions of cell death. Annu. Rev. Cell Biol. 7, 663-698.

Ellis, M. C., O'Neill, E. M. and Rubin, G. M. (1993). Expression of Drosophila glass protein and evidence for negative regulation of its activity in non-neuronal cells by another DNA-binding protein. Development 119, 855-865.

Evan, G. I., Wyllie, A. H., Gilbert, C. S., Littlewood, T. D., Land, H., Brooks, M., Waters, C. M., Penn, L. Z. and Hancock, D. C. (1992). Induction of apoptosis in fibroblasts by c-myc protein. Cell 69, 119-128. 
Fairbairn, L. J., Cowling, G. J., Reipert, B. M. and Dexter, T. M. (1993) Suppression of apoptosis allows differentiation and development of a multipotent hemopoietic cell line in the absence of added growth factors. Cell 74, 823-832.

Glucksmann, A. (1950). Cell deaths in normal and vertebrate ontogeny. Biol. Rev. Cambridge Philos. Soc. 26, 59-86.

Gooding, L. R., Elmore, L. W., Tollefson, A. E., Brady, H. A. and Wold, W. S. M. (1988). A 14,700 MW protein from the E3 region of adenovirus inhibits cytolysis by tumor necrosis factor. Cell 53, 341-346.

Gooding, L. R., Ranheim, T. S., Tollefson, A. E., Aquino, L., DuerksenHughes, P. J., Horton, T. M. and Wold, W. S. M. (1991). The 10,400- and 14,500-dalton proteins encoded by region E3 of adenovirus function together to protect many but not all mouse cell lines against lysis by tumor necrosis factor. J. Virol. 65, 4114-4123.

Gooding, L. R., Aquino, L., Duerlsen-Hughes, P. J., Day, D., Horton, T. M., Yei, S. and Wold, W. S. M. (1991). The E1B-19K protein of group C adenoviruses prevents cytolysis by tumor necrosis factor of human cells but not mouse cells. J. Virol. 65, 3083-3094.

Gregory, C. D., Dive, C., Henderson, S., Smith, C. A., Williams, G. T., Gordon, J. and Rickson, A. B. (1991). Activation of Epstein-Barr virus latent genes protects human B cells from death by apoptosis. Nature 349, 612-614.

Hashimoto, S., Ishii, A. and Yonehara, S. (1991). The E1B oncogene of adenovirus confers cellular resistance to cytotoxicity of tumor necrosis factor and monoclonal anti-Fas antibody. Int. Immunol. 3, 343-351.

Hay, B. A., Ackerman, L., Barbel, S., Han, L. and Jan, Y. N. (1988). Identification of a component of Drosophila polar granules. Development 103, 625-640

Henderson, S., Rowe, M., Gregory, C., Croom-Carter, D., Wang, F., Longnecker, R., Kieff, E. and Rickinson, A. (1991). Induction of bcl-2 expression by Epstein-Barr virus latent membrane protein 1 protects infected B cells from programmed cell death. Cell $\mathbf{6 5}, 1107-1115$.

Henderson, S., Huen, D., Rowe, M., Dawson, C., Johnson, G. and Rickson, A. (1993). Epstein-Barr virus-coded BHRF1 protein, a viral homologue of Bcl-2, protects human B cells from programmed cell death. Proc. Natl. Acad. Sci. USA 90, 8479-8483.

Hiromi, Y. and Gehring, W. J. (1987). Regulation and function of the Drosophila segmentation gene fushi tarazu. Cell 50, 963-974.

Levine, B., Huang, Q., Isaacs, J. T., Reed, J. C., Griffin, D. E. and Hardwick, J. M. (1993). Conversion of lytic to persistent alpha-virus infection by the bcl-2 cellular oncogene. Nature 361, 739-742.

Lowe, S. W., Schmitt, E. M., Smith, S. W., Osborne, B. A. and Jacks, T. (1993). p53 is required for radiation-induced apoptosis in mouse thymocytes. Nature 362, 847-849.

Martz, E. and Howell, D. M. (1989). CTL: virus control cells first and cytolytic cells second? Immunol. Today 10, 79-86.

Meyaard, L., Otto, S. A., Jonker, R. R., Mijnster, M. J., Keet, P. M. and Miedema, F. (1992). Programmed death of T cells in HIV-1 infection. Science 257, 217-219.

Moses, K., Ellis, M. C. and Rubin, G. M. (1991). glass encodes a site-specific DNA-binding protein that is regulated in response to positional signals in the developing Drosophila eye. Genes and Devel. 5, 583-593.
Murphy, K. M., Heimberger, A. B. and Loh, D. Y. (1990). Induction by antigen of intrathymic apoptosis of $\mathrm{CD} 4+\mathrm{CD} 8+\mathrm{TCR}^{\circ}$ thymocytes in vivo. Science 250, 1720-1723.

Pilder, S., Logan, J. and Shenk, T. (1984). Deletion of the gene encoding the adenovirus 5 early region 1B - 21,000-molecular weight polypeptide leads to degradation of viral and cellular DNA. J. Virol. 52, 664-671.

Pirotta, V. (1988). In Vectors: A Survey of Molecular Cloning Vectors and their Uses. (eds. Rodriguez, R. L. and Denhardt, D. T.) pp. 437-456. Boston: Butterworths.

Rabizadeh,S., LaCount, D. J., Friesen, P. D. and Bredesen, D. E. (1993) Expression of the baculovirus p35 gene inhibits mammalian cell death. $J$. Neurochem. 61, 2318-2321.

Raff, M. C. (1992). Social control on cell survival and cell death. Nature 356, 397-400.

Rubin, G. M. and Spradling, A. C. (1982). Genetic transformation of Drosophila with transposable element vectors. Science $\mathbf{2 1 8}, 348-353$.

Spreij, TH. E. (1971). Cell death during the development of the imaginal discs of Calliphora erythrocephalia. Netherlands J. Zool. 21, 221-264.

Subramanian, T., Kuppuswamy, M., Gysbers, J., Mak, S. and Chinnadurai, G. (1984). kDa tumor antigen coded by early region E1b of adenovirus 2 is required for efficient synthesis and for protection of viral DNA. J. Biol. Chem. 259, 11777-11783.

Sugimoto, A., Friesen, P. D. and Rothman, J. H. (1994). Baculovirus p35 prevents developmentally programmed cell death and rescues a ced-9 mutant in the nematode Caenorhabditis elegans. EMBO J. 13, 2023-2028.

Vaux, D. L. (1993). Toward an understanding of the molecular mechanisms of physiological cell death. Proc. Natl. Acad. Sci. USA. 90, 786-789.

White, E. and Stillman, B. (1987). Expression of the adenovirus E1B mutant phenotypes is dependent on the host cell and on the synthesis of E1A proteins. J. Virol. 61, 426-435.

White, E., Sabbatini, P., Debbas, M., Wold, W. S. M., Kusher, D. I. and Gooding, L. R. (1992). The 19-kilodalton adenovirus E1B transforming protein inhibits programmed cell death and prevents cytolysis by tumor necrosis factor alpha. Mol. Cell. Biol. 12, 2570-2580.

Winberg, M. L., Perez, S. E. and Steller, H. (1992). Generation and early differentiation of glial cells in the first optic ganglion of Drosophila melanogaster. Development 115, 903-911.

Wolff, T. and Ready, D. F. (1991). Cell death in normal and rough eye mutants of Drosophila. Development 113, 825-839.

Wolff, T. and Ready, D. F. (1993). Pattern formation in the Drosophila retina. In The Development of Drosophila melanogaster. (eds. M. Bate, M. and A. Martinez Arias). Cold Spring Harbor Laboratory Press.

Wyllie, A. H., Kerr, J. F. R. and Currie, A. (1980). Cell death: the significance of apoptosis. Int. Rev. Cytol. 68, 251-306.

Yonish-Rouach, E., Resnitzky, D., Lotem, J., Sachs, L., Kimchi, A. and Oren, M. (1991). Wild-type p53 induces apoptosis of myeloid leukaemic cells that is inhibited by interleukin-6. Nature 352, 345-347. 